SANTOS, A.L.Q. et al. Abordagem cirúrgica da articulação coxofemoral de ouriço-cacheiro (Coendou prehensilis, Linnaeus, 1758) (Rodentia). PUBVET, Londrina, V. 5, N. 18, Ed. 165, Art. 1117, 2011.

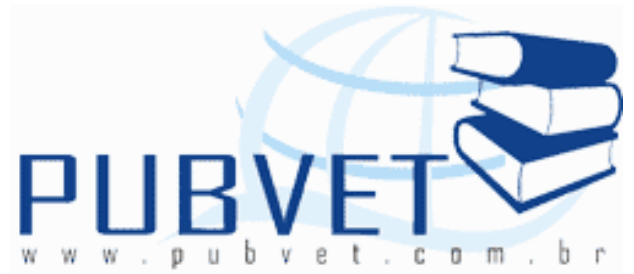

PUBVET, Publicações em Medicina Veterinária e Zootecnia.

\title{
Abordagem cirúrgica da articulação coxofemoral de ouriço-cacheiro (Coendou prehensilis, Linnaeus, 1758) (Rodentia)
}

André Luiz Quagliatto Santos ${ }^{1}$, Simone Borges Salgueiro De Simone ${ }^{2}$, Saulo Fernandes Mano de Carvalho ${ }^{1}$, Lorena Tannús Menezes ${ }^{2}$, Liliane Rangel Nascimento ${ }^{2}$, Arthur Paulino Sanzo Kaminishi ${ }^{2}$, Juliana Macedo Magnino Silva ${ }^{2}$ Laboratório de Ensino e Pesquisas em Animais Silvestres - LAPAS, FAMEV/UFU, e-mail: quagliatto@famev.ufu.br 1. Docentes. 2. Mestrandos.

\section{Resumo}

Objetivou-se estudar a técnica de abordagem cirúrgica da articulação coxofemoral de ouriço-cacheiro (Coendou prehensilis), utilizando quatro exemplares adultos que foram encaminhados ao Laboratório de Ensino e Pesquisa em Animais Silvestres. Uma incisão de pele sobre o trocânter maior do fêmur, ao longo da borda cranial do músculo bíceps femoral, a fim de liberar o músculo tensor da fáscia lata com sua borda no glúteo médio foi a técnica de escolha. As margens cutâneas foram divulsionadas e afastadas. A técnica mostrou-se efetiva para o animal em questão.

Palavras-chave: Anatomia, membro pélvico, luxação 
SANTOS, A.L.Q. et al. Abordagem cirúrgica da articulação coxofemoral de ouriço-cacheiro (Coendou prehensilis, Linnaeus, 1758) (Rodentia). PUBVET, Londrina, V. 5, N. 18, Ed. 165, Art. 1117, 2011.

\title{
Surgical approach to the hip joint of hedgehog (Coendou prehensilis, Linnaeus, 1758) (Rodentia)
}

\begin{abstract}
The objective was to study the technique of surgical approach to the hip joint of hedgehog (Coendou prehensilis) using four adult specimens that were sent to the Laboratory for Teaching and Research on Wild Animals. A skin incision over the greater trochanter, along the cranial border of the biceps femoris muscle in order to release the muscle tensor fascia lata with its edge in the gluteus medius was the technique of choice. The skin margins were divulsed and remote. The technique proved effective for the animal in question.
\end{abstract}

Keywords: Anatomy, pelvic limb, dislocation

\section{INTRODUÇÃO}

Devido ao crescimento desordenado das áreas urbanas, os animais silvestres, principalmente os mamíferos, são vistos em acostamentos de rodovias vitimas de atropelamento ou encontrados quase em óbito por ataques de cães. Nestes casos, uma das patologias mais observadas são as fraturas.

Assim como cães e gatos, estes animais também são encaminhados com freqüência a clínicas veterinárias apresentando fraturas. Em cães foi reportado que fraturas proximais na epífise do osso fêmur ocorrem em 16\% de todas as fraturas nas epífises dos ossos de cães jovens (MARRETTA E SCHRADER, 1983).

A articulação coxofemoral é freqüentemente o local mais acometido pela luxação traumática em cães e gatos. De acordo com BRINKER et al. (1999), representa cerca de $50 \%$ de todas as artropatias por deslocamento.

EATON-WELLS e WHITTICK (1990), BRINKER et al. (1999) e JOHNSTON (1999) relatam que a luxação coxofemoral pode ser crânio-dorsal, caudodorsal e ventral, podendo haver rompimento da cápsula articular e do ligamento redondo. 
SANTOS, A.L.Q. et al. Abordagem cirúrgica da articulação coxofemoral de ouriço-cacheiro (Coendou prehensilis, Linnaeus, 1758) (Rodentia). PUBVET, Londrina, V. 5, N. 18, Ed. 165, Art. 1117, 2011.

As técnicas de redução aberta incluem capsulorrafia, colocação de pino transacetabular, introdução de pino de Vita ou pino de Toggle, transposição trocantérica, uso de prótese e aplicação de fixador externo flexível (LIPOWITZ et al. 1993; FOSSUM, 1997; JHNSTON, 1999).

Nayak et al. (2001) atenderam 15 cães e seis gatos com fraturas, sendo seis fraturas de úmero, sete de rádio e ulna, cinco de rádio, ulna e metacarpo, três fraturas cominutivas de rádio e ulna, sete de fêmur, dez de tíbia e fíbula, seis de tarso e cinco fraturas cominutivas de tíbia e fíbula. Fraturas proximais na epífise do osso fêmur também ocorrem em outras espécies, incluindo o homem (CRAWFORD, 1998; WILSON et al.1991).

A finalidade no tratamento de uma fratura é obter rápida união óssea, preservando a função normal das articulações e tecidos moles próximos. O conhecimento anatômico de cada espécie fornece subsídios para realização de procedimentos cirúrgicos com segurança, assim objetivou-se estudar a técnica de abordagem cirúrgica da porção média do fêmur de Coendou prehensilis.

\section{MATERIAL E MÉTODO}

Utilizou-se quatro Coendou prehensilis adultos, sendo 3 machos e 1 fêmea, provenientes do Zoológico Parque do Sabiá, Uberlândia-M.G., após mortes naturais. As espécimes foram enviadas ao Laboratório de Ensino e Pesquisa em Animais Silvestres (LAPAS), onde as a artéria aorta torácica foi canulada e preenchida com solução corada de Neoprene Látex "450" ®. A veia jugular também foi canulada e preenchida com solução de formol a $10 \%$. A seguir, o material foi fixado em solução de formol a $10 \%$ injetado por via intramuscular profunda, subcutânea e intravicatária, permanecendo submersos nesta solução por um período mínimo de 48 horas.

Retirou-se a pele do membro pelvino a fim de visualizar a musculatura da pelve e coxa. Os músculos pélvicos foram dissecados, onde se observou suas origens e inserções. A articulação coxofemoral foi acessada de modo a 
SANTOS, A.L.Q. et al. Abordagem cirúrgica da articulação coxofemoral de ouriço-cacheiro (Coendou prehensilis, Linnaeus, 1758) (Rodentia). PUBVET, Londrina, V. 5, N. 18, Ed. 165, Art. 1117, 2011.

diminuir as incisões e traumas cirúrgicos através da seguinte manobra: incisão da pele centrada no trocânter maior do fêmur, situado pela borda distal da diáfise femoral, com divulsão e afastamento das margens cutâneas. Fez-se uma incisão através do músculo glúteo superficial, ao longo da borda cranial dos músculos reto femoral e vasto lateral. O músculo glúteo médio foi afastado dorsalmente e os músculos reto femoral e vasto lateral foram afastados caudalmente. Assim alcançou-se e incindiu-se a cápsula articular, expondo a articulação coxofemoral.

\section{RESULTADOS E DISCUSSÃO}

Para acessar a articulação coxofemoral de cães BRANDÃO, IAMAGUTI e FIGUEIREDO (2002), utilizaram a técnica descrita por PIERMATTEI, D.C., (1993), realizando uma incisão de pele sobre o trocânter maior do fêmur, situado sobre a borda cranial da diáfise femoral e uma incisão na fáscia lata, e músculo tensor da fáscia lata, ao longo da borda cranial do músculo bíceps femoral, a fim de liberar o músculo tensor da fáscia lata com sua borda no glúteo médio.

Para o acesso dorso-lateral da articulação coxofemoral de cão IAMAGUTI et al.(1994) utilizou o acesso entre o trocânter maior e as apófises das vértebras sacrais. BERZON et al. (1980), utilizou tenotomia nos músculos glúteo superficial e glúteo médio. TOMLINSON e MACCAUGHLIN, 1996, incindiu o bíceps femoral em um retalho crânio-caudal e EVERS et al. (1997) divulsionou o músculo bíceps e a fáscia lata.

De um modo geral as técnicas utilizadas pelos autores para acessar a articulação coxofemoral são semelhantes à descrita por PIERMATTEI, D.C. (1993) assim como no presente estudo, no entanto tal técnica foi adaptada a anatomia do Coendou prehensilis. Mesmo com as adaptações tal técnica mostrou-se pouco traumática, sendo acessada com o mínimo de incisões, principalmente em origens e inserções dos músculos, elegendo sempre a divulsão muscular em detrimento da incisão. 
As observações mais expressivas na anatomia de C. prehensilis foi a ausência de fáscia lata, músculo tensor da fáscia lata cranial ao reto femoral e vasto lateral. Centrando a incisão no trocânter maior do fêmur do ouriçocacheiro, identificou-se os seguintes níveis teciduais: pele, subcutâneo, músculos glúteo superficial e glúteo profundo, pelve e articulação coxofemoral.

Para aumentar a área de acesso, os músculos reto femoral e vasto lateral são afastados caudalmente. Esta espécie apresenta músculos pélvicos com origens mais caudais em relação aos animais domésticos, sendo esta uma característica de suma importância a ser observada.

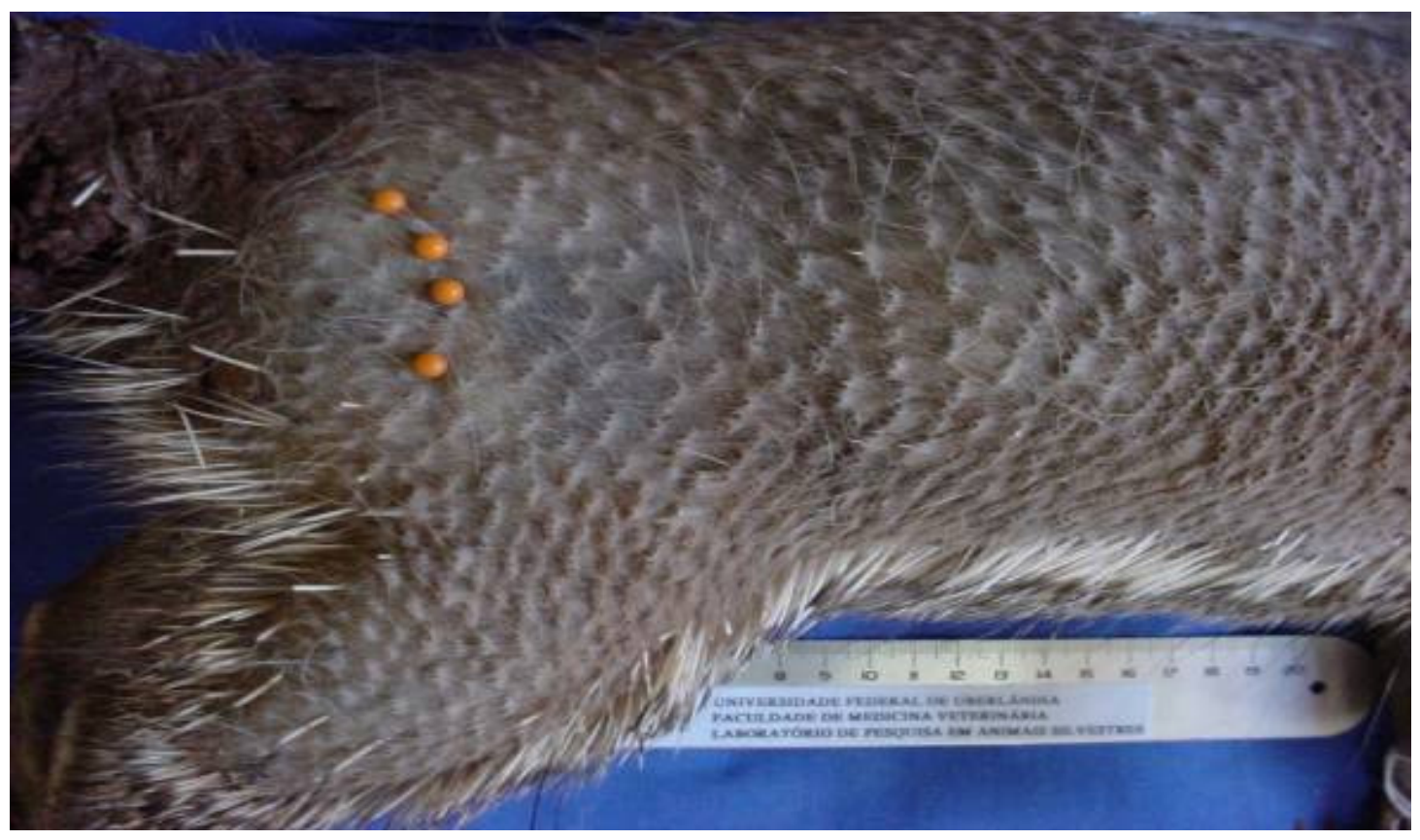

FIGURA 1: Vista lateral do membro pélvico de $C$. prehensilis. Os alfinetes marcam o local de incisão para acessar à articulação coxo femoral. 


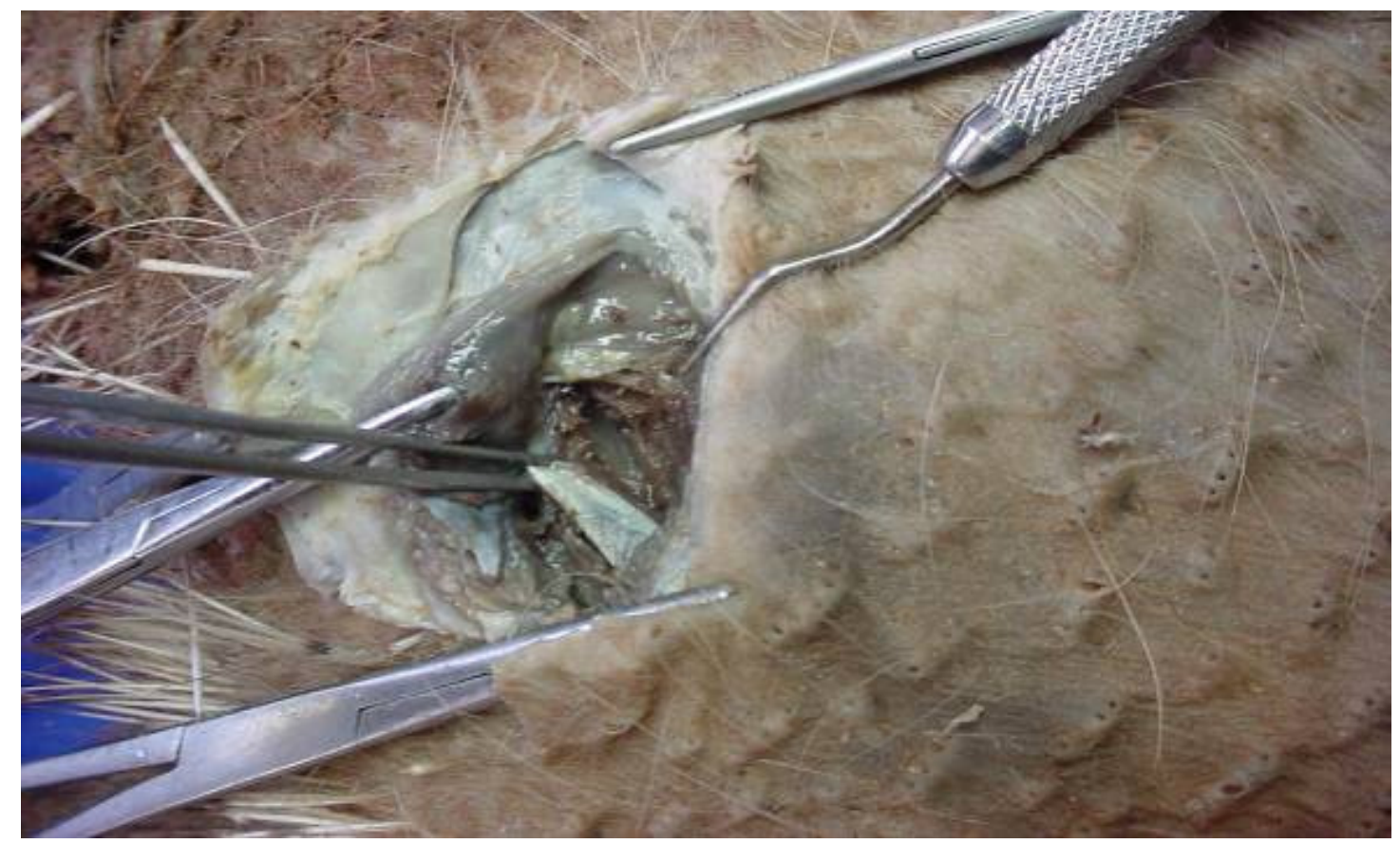

FIGURA 2: Face lateral do membro pélvico de $C$. prehensilis onde se observa o acesso à articulação coxofemural.

\section{CONCLUSÃO}

Constatou-se que é possível o acesso a articulação coxofemoral de Coendou prehensilis através de uma técnica de abordagem simples e pouco traumática.

\section{REFERÊNCIAS}

BERZON, J.L.; HOWARD, P.E.; COVELL, S.J.; TROTTER, E.J.; DUELAND, R.A. A restrospective study of the efficacy of femoral head and neck excisions in 94 dogs and cats. Veterinary Surgery. V,9, n.3, p. 88-92, 1980.

BRANDÃO, C.V.S.; IAMACUTI, P.; FIGUEIREDO, L.M.A. Substituição do ligamento da cabeça do fêmur com auto-enxerto de fáscia lata na luxação coxofemoral em cães. Ciência Rural, V. 32, n. 2, 2002.

BRINKER, W.O.; PIERMATTEI, D.L.; FLO, G.L. A articulação coxofemoral. In: Manual de Ortopedia e Tratamento das Fraturas dos Pequenos Animais. São Paulo: Manole, 1999. p. 394-436.

EATON-WELLS, R.; WHITTICK, W.G. Luxations. In: WHITTICK, W.G. Canine Orthopedics. 2 ed. Philadelphia: Lea e Febiger, 1990. p. 491-518. 
EVERS, P.; KRAMER, B.A.; WALLACE, L.J.; JOHNSTON, G.R.; KING, V. Clinical and radiografic evaluation of intertrochanteric ostrotomy in dogs: a retrospective study of 18 dogs. Am. College. Veterinary Surgery, v. 26, p. 217-222, 1997.

FOSSUM, T.W. Management of joint disease. In: Small Animal Surgery. St. Louis: Mosby, 1997. p. 883-998.

IAMAGUCHI, P.; DEL CARLO, R.J.; VULCANO, L.C.; GANDOLI, W.; TEIXEIRA, R.C. Excissão artro plástica da cabeça do fêmur de cães. In: Aspectos cirúrgicos e histopatológicos. Vet. Zoot., v.6, p. 43-55, 1994.

JOHNSTON, S. A. Articulações. In: HARARI, J. Cirurgia de Pequenos Animais. Porto Alegre: Artmed, 1999. p. 287-312.

LIPOWITS,A.T.; CAYWOOD, D.D.; NEWTON, C.D.; FINCH, M.E. Coxofemoral joint. In: Small Animal Orthopedics Illustrated. St. Louis: Mosby, 1993. p. 189-216.

PIERMATTEI, D.L. An atla of surgical approaches to the bones and joints of the dog and cats. 3 ed. Philadelphia: Sawnders, 1997. Cap. 15: The hip joint: p. 422-468.

RODASKI, S.; CUNHA, O.; DE NARDI, A.B.; RIOS, A.; COMAR, F.A.; CASTRO, J.H.T. Artroplastia acetábulo- femoral em cães com pericárdio bovino conservado. Archives o Veterinary Science, v.7,n.2, 2002.

TOMLINSON, J.; MCCAUGHIN, R. Total hip rephacement: the best treatment for dysplastic dogs with orteoarthosis. Vet. Med., v.91, n.2, p. 118-124, 1996. 